Discussion Papers of the

Max Planck Institute for

Research on Collective Goods

2020/6

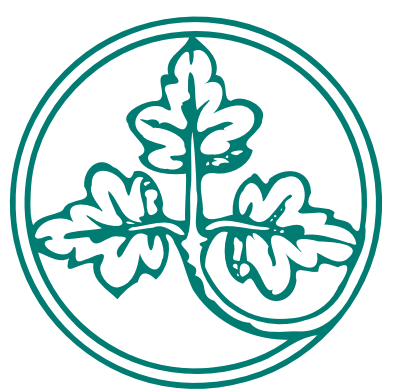

Rechtswissenschaft als

Sozialwissenschaft

Spurensuche im Jahrgang 2019 der ZGR

Christoph Engel 


\section{Rechtswissenschaft als Sozialwissenschaft Spurensuche im Jahrgang 2019 der ZGR}

Christoph Engel

February 2020 


\section{Einleitung}

Es gibt einen Grund, warum das Fach Jurisprudenz heißt und nicht Jurisscienz. Juristen müssen entscheiden. Wenn sie zu der Überzeugung gelangen, dass ein Gesichtspunkt für diese Entscheidung wichtig ist, dürfen sie den Gesichtspunkt nicht ignorieren. Das gilt auch dann, wenn dieser Gesichtspunkt noch nicht vollständig verstanden ist. Ein normatives Anliegen darf nicht mit der Begründung verworfen werden, dass seine theoretische Grundlage umstritten ist. Wenn die Sorge um einen normativ unerwünschten Effekt besteht, darf der Effekt nicht mit der Begründung außeracht gelassen werden, dass er unerforscht oder noch nicht verlässlich genug bewiesen ist. Potentiell muss das Recht auch auf unsicherer konzeptioneller oder tatsächlicher Grundlage entscheiden. Das ist der Grund, warum das deutsche Recht, anders als das amerikanische, vom Richter kein Wahrscheinlichkeitsurteil erwartet, sondern eine persönliche Überzeugung (§ 286 I 2 ZPO).

Hinter dem Streit zwischen den Rechtsordnungen auf dem europäischen Kontinent und dem common law über das Beweismaß steht eine Divergenz zwischen zwei regulativen Ideen ${ }^{1}$. Wer Wahrscheinlichkeitsurteile fordert, will die Rechtsprechung so weit als irgend möglich objektivieren. Wer dagegen auf die persönliche Überzeugung der Person setzt, der das Amt des Richtens anvertraut ist, der will nach Möglichkeit dem konkreten Fall gerecht werden. Hinter den konkurrierenden regulativen Ideen steht eine unterschiedliche Definition des regelungsbedürftigen Problems ${ }^{2}$. Wahrscheinlichkeitsurteile sind angemessen, wenn die Eigenheiten eines Falles vernachlässigt werden können, die der notwendigen Standardisierung zum Opfer fallen. Die persönliche Überzeugung ist dagegen gefordert, wenn man nicht an die Standardisierbarkeit der Fälle des Lebens glaubt. Das französische Recht bringt diese Position besonders eindringlich mit der Instruktion zum Ausdruck, die Strafrichter erhalten. Sie werden aufgefordert, nach Maßgabe ihrer „intime conviction“ zu entscheiden ${ }^{3}$.

In diesem Streit der Rechtsordnungen habe ich mich auf die Seite meiner eigenen Rechtsordnung geschlagen. Ich bin überzeugt, dass juristische Fälle nicht nur ausnahmsweise, sondern regelmäßig „ill defined“ sind. Deshalb kann die Entscheidung nicht allein mit den Mitteln der Logik aus konsentierten normativen Prinzipien abgeleitet werden kann ${ }^{4}$. Deshalb glaube ich nicht, dass die Entscheidungen von Richtern vollständig formalisiert und modelliert werden können. Allenfalls kann man (in einem „satisficing“ Ansatz) äußerste Grenzen bestimmen, die die Entscheidung im Einzelfall nicht überschreiten darf ${ }^{5}$.

Heißt das, dass auch die Rechtswissenschaft lieber Geisteswissenschaft bleiben und die hermeneutische Kunst der Auslegung des Gesetzes verfeinern sollte? Die Dogmatik wird der Kern der Disziplin bleiben. Aber auch eine Rechtswissenschaft, die ihre vornehmste Aufgabe im Dienst an der Rechtspraxis sieht, kann deshalb doch mit Gewinn sozialwissenschaftliche Methoden verwenden. Der Dienst besteht dann in der kritischen Überprüfung

1 C. Engel, Preponderance of the Evidence Versus Intime Conviction. A Behavioral Perspective on a Conflict Between American and Continental European Law, Vermont Law Review 33 (2009), 435-467.

2 L. Daston, Objectivity versus Truth,Bödeker u.a. Wissenschaft als kulturelle Praxis, 1750-1900, 17-32.

$3 \quad$ Art. 353 Code de Procédure Pénale.

4 S. zu dem Komplement des Problems auf der Seite der normativen Theorie C. Engel, Offene Gemeinwohldefinitionen, Rechtstheorie 32 (2001), 23-52.

5 C. Engel/W. Güth, Modeling a Satisficing Judge, Rationality and Society 30 (2018), 220-246. 
normativer Argumente, die für rechtsdogmatische oder rechtspolitische Entscheidungen hinreichend wichtig sind. Der Rechtswissenschaftler entscheidet dann allerdings nicht mehr selbst. Er liefert aber eine sehr viel belastbarere Entscheidungsgrundlage. Im Ergebnis können die Rechtsanwender lernen, dass bestimmte Argumente ganz falsch sind, viel zweifelhafter als man glaubte, oder bloß spekulativ. Umgekehrt können sozialwissenschaftliche Methoden auf normative Belange aufmerksam machen, die bislang keinen oder zu wenig Einfluss auf die Entscheidung hatten. Wenn Rechtswissenschaft sozialwissenschaftlich arbeitet, wird die Arbeitsteilung zwischen Rechtsanwendung und Rechtswissenschaft ausgeprägter. Aber stärkere Arbeitsteilung erhöht die Chance auf bessere Entscheidungen.

Das Gesellschaftsrecht eignet sich für diese Art von Arbeitsteilung besonders gut. Sein Gegenstand ist die Organisation und das Funktionieren von Unternehmen. Unternehmen sind juristische Zweckschöpfungen. Die Erzielung von Gewinn ist typischerweise zumindest einer dieser Zwecke. Dieser Gegenstand führt zu einer Nähe zu den Wirtschaftswissenschaften. Auch in der historischen Entwicklung ist diese Nähe zu spüren. Das Gesellschaftsrecht hat sich für ökonomische Argumente viel früher geöffnet als andere Teilrechtsgebiete. Deshalb eignet sich das Gesellschaftsrecht besonders gut für die Art von Spurensuche, die dieser Aufsatz beabsichtigt. Sozialwissenschaftliche Methoden schaffen Distanz zur Rechtspraxis. Spuren dieser Methoden sind deshalb eher in einer Archivzeitschrift zu erwarten. Sozialwissenschaftliche Argumente im Gesellschaftsrecht haben zwar eine lange Tradition ${ }^{6}$. Die ökonomische Analyse des Rechts hat jedoch erst in den siebziger Jahren Fahrt aufgenommen ${ }^{7}$. Das empirical legal movement ist kaum mehr als ein Jahrzehnt alt. ${ }^{8}$ Deshalb sind in jüngeren Publikationen mehr Spuren zu erwarten als in älteren. Diese Überlegungen motivieren die Wahl des Jahrgangs 2019 der ZGR als den Ort für die Spurensuche.

\section{Das Material}

Der Jahrgang 2019 der Zeitschrift für Unternehmens- und Gesellschaftsrecht ZGR enthält auf 1170 Seiten 31 Aufsätze von 32 Autoren. Vier Aufsätze haben zwei Autoren. Zwei Autoren (Klaus Hopt und Patrick Leyens) haben einen gemeinsamen und je noch einen weiteren Aufsatz als Alleinautor veröffentlicht. 26 Autoren sind Universitätsprofessoren, fünf sind Rechtsanwälte. Ein promovierter Autor habilitiert sich (Korch). Der Koautor eines Professors ist noch nicht promoviert (Bialluch). Zwei Professoren lehren in Österreich (Doralt, Leyens) und je einer in Belgien (Cools) und Luxemburg (Zetzsche). Die übrigen Autoren lehren an deutschen Universitäten oder Max Planck Instituten. Vier Professoren sind emeritiert (Beuthien, Hommelhoff, Hopt, Wiedemann). Sechs Autoren berichten auf ihren Websites, dass sie auch eine ökonomische Ausbildung haben (Anzinger, Bueren, Fleischer, Hopt, Schön, Thole). Alle sechs sind Universitätsprofessoren. 
Sieben Texte sind Urteilsanmerkungen, der Rest sind Archivaufsätze. 25 Aufsätze sind einem Thema der Corporate Governance gewidmet. Die größte Aufmerksamkeit gilt der Aktiengesellschaft (22 Aufsätze). Mit Themen aus dem Recht der $\mathrm{GmbH}$ beschäftigen sich 6 Aufsätze, genauso viele mit dem Recht der Personengesellschaft. Ein Aufsatz hat das Stiftungsrecht zum Gegenstand. Das größte Interesse gilt dem Konzernrecht (11 Aufsätze), gefolgt vom Vorstand einer Aktiengesellschaft (8 Aufsätze) und vom Aufsichtsrat (7 Aufsätze). Fünf Aufsätze gelten dem Kapitalmarktrecht. Vier haben prozessrechtliche Fragen zum Gegenstand.

21 Aufsätze sind im engeren Sinne dogmatischer Natur, widmen sich also der Auslegung einer oder mehrerer Normen des geltenden Gesellschaftsrechts. 18 Aufsätze haben jedenfalls auch eine rechtspolitische Ausrichtung. 20 Aufsätze möchten ein normatives Leitbild formen. Drei Aufsätze zeichnen die historische Entwicklung nach, sechs Aufsätze berichten über politische Entwicklungen.

\section{Sozialwissenschaftliche Forschungsfragen}

Wenn man den sozialwissenschaftlichen Charakter eines Textes eng definiert, sucht man im Jahrgang 2019 der ZGR vergebens. Kein Text entwickelt ein formales Modell, oder nutzt Daten, um einen kausalen Effekt zu identifizieren.

Ein Aufsatz sticht jedoch heraus. Katja Langenbucher umreißt das Potenzial für eine kognitive Wende im Gesellschaftsrecht. Sie macht die Juristen auf die Bedeutung der Verhaltenswissenschaften für das Design und die Interpretation gesellschaftsrechtlicher Institutionen aufmerksam. Sie greift dafür weit aus. Sie berichtet nicht nur über Relativierungen und Verfeinerungen des klassischen mikroökonomischen Modells. Sie tastet auch die Bedeutung von Sozial- und Organisationspsychologie ab und greift sogar auf die Neurowissenschaften aus ${ }^{9}$. Auch in den Sozialwissenschaften haben solche Überblicksaufsätze ihren Platz. Sie verschaffen Orientierung und machen auf Forschungslücken aufmerksam.

Vier weitere Aufsätze kann man als Transferleistungen beschreiben. Sie dokumentieren den Stand der Diskussion in einer Sozialwissenschaft und machen deutlich, in welcher Weise das Gesellschaftsrecht daraus Nutzen ziehen kann. Alle vier Aufsätze beziehen sich dabei auf die Wirtschaftswissenschaften.

Eckart Bueren bezieht sich in seinem Aufsatz zum Konzept der nachhaltigen Finanzierung vor allem auf betriebswirtschaftliche Literatur. Diese Literatur hilft ihm zunächst, seinen Gegenstand zu definieren und seine wirtschaftliche Bedeutung einzuschätzen. Aus dieser Literatur entnimmt er eine für die rechtliche Einordnung bedeutsame Unterscheidung: ist die Rücksicht auf Nachhaltigkeit der beste Weg zum unternehmerischen Erfolg, oder gibt es

$9 \quad$ K. Langenbucher, Interdisziplinäre Forschung im Unternehmensrecht-auf dem Weg zu einer cognitive corporate governance?, Zeitschrift für Unternehmens-und Gesellschaftsrecht 48 (2019), 717-759. 
einen Zielkonflikt zwischen dem Gewinnstreben und der Rücksicht auf konkurrierende normative Belange? Die eigentliche Forschungsfrage ist dann aber genuin juristischer Natur: auf welche Weise kann und sollte nachhaltige Finanzierung rechtlich gesichert werden ${ }^{10}$ ?

Tobias Tröger nutzt den konzeptionellen Apparat von law and economics, um Anliegen der neuen Aktionärsrechterichtlinie besser zu verstehen und Vorschläge für die geeignete Umsetzung in das deutsche Recht zu machen. Typischerweise liegen die Aktien börsennotierter Unternehmen bei Anlegern, die wenig Anreiz haben, die Verwaltung des Unternehmens wirksam zu kontrollieren. Das konstituiert ein Prinzipal-Agent-Problem. Herrschaft und Haftung fallen tendenziell auseinander. Dieser Konflikt wird durch die Zwischenschaltung von institutionellen Anlegern und Vermögensverwaltern verschärft. Im Grundsatz führt das zu einem Marktversagen. Regulatorische Interventionen sind gerechtfertigt. Ob die Verpflichtung institutioneller Anleger auf „Stewardship“ dem abhelfen kann, erscheint Tobias Tröger jedoch zweifelhaft ${ }^{11}$.

Auch Tim Florstedt interessiert sich für die Umsetzung der Aktionärsrechterichtlinie. Er konzentriert sich jedoch auf die Vorschriften über die Vergütung von Mitgliedern des Vorstands und des Aufsichtsrats einer Aktiengesellschaft. Er rezipiert die ökonomische Literatur zu Fehlanreizen für diese Organwalter. Aus diesen Konzeptualisierungen folgt allerdings nicht viel für die anschließende dogmatische Analyse ${ }^{12}$.

Klaus Ulrich Schmolke diskutiert Rechtsregeln für Whistleblower. Zur Analyse der regulatorischen Entwicklung greift er auf betriebswirtschaftliche und psychologische Literatur zurück. Aus der rechtsökonomischen Literatur zu einer expressiven Funktion von Rechtsregeln entnimmt er einen zusätzlichen Wirkungskanal: explizite rechtliche Regeln können die gesellschaftliche Einstellung gegenüber Whistleblowern verändern. Im Zentrum der Analyse steht dann aber das Anreizproblem: warum sollte sich jemand zum Wohle des gesamten Unternehmens oder der Allgemeinheit Risiken am Arbeitsplatz aussetzen? Deshalb bedarf es nicht nur des Schutzes, sondern grundsätzlich auch positiver Anreize. Sie müssen allerdings in ein Gleichgewicht gegenüber dem Missbrauchspotenzial gebracht werden ${ }^{13}$.

\section{Literarische Spuren}

Bei sechs weiteren Aufsätzen muss man die Fußnoten schon genau lesen, um die literarischen Spuren aus den Sozialwissenschaften zu entdecken.

\footnotetext{
10 E. Bueren, Sustainable Finance, Zeitschrift für Unternehmens-und Gesellschaftsrecht 48 (2019), 813-875.

$11 T$. Tröger, Die Regelungen zu institutionellen Investoren, Vermögensverwaltern und Stimmrechtsberatern im Referentenentwurf eines Gesetzes zur Umsetzung der zweiten Aktionärsrechterichtlinie (ARUG II), Zeitschrift für Unternehmens-und Gesellschaftsrecht 48 (2019), 126-162.

12 T. Florstedt, Die neuen Aktionärsvoten zur Organvergütung, Zeitschrift für Unternehmens- und Gesellschaftsrecht 48 (2019), 630-663.

13 K. U. Schmolke, Whistleblowing als Regelungsaufgabe, Zeitschrift für Unternehmens- und Gesellschaftsrecht 48 (2019), 876-922.
} 
Wolfgang Schön begründet die Regelung der Rechtsbeziehungen im Konzern mit der Einsicht von Ronald Coase, dass Märkte nicht der beste institutionelle Rahmen für die Allokation knapper Güter sind, wenn die Transaktionskosten prohibitiv hoch wären. Deshalb widmet sich der Aufsatz den institutionellen Anforderungen an eine effiziente Allokation und vor allem der richtigen Balance zwischen dem Schutz der abhängigen Gesellschaft und dem Gruppeninteresse ${ }^{14}$.

Birgit Weitemeyer begründet die Notwendigkeit von Rechtsregeln für die governance von Stiftungen mit der klassischen Einsicht von Berle und Means, dass die Herrschaft (ausgeübt von den Stiftungsorganen) und Haftung (durch den Stifter) auseinanderfallen ${ }^{15}$.

Heribert Anzinger interessiert sich für die Regeln über die Vergütung von Mitgliedern des Vorstands und des Aufsichtsrats. Er zitiert ein ökonomisches Diskussionspapier, das die Gründe für den Anstieg der Vergütungen erörtert ${ }^{16}$.

Hans Christoph Grigoleit ist an den Regeln der Aktionärsrechterichtlinie über Geschäfte mit nahestehenden Personen interessiert. Er untersucht vor allem das Verhältnis zum deutschen Konzernrecht. Zur Beschreibung des normativen Problems verweist er auf den in der ökonomischen Literatur verwendeten Begriff des Tunneling, und zitiert dazu einen Text, der in der American Economic Review erschienen ist ${ }^{17}$.

Klaus Hopt und Patrick Leyens analysieren den Corporate Governance Code 2020. Als Teil des reichen Materials zitieren sie einen Überblicksaufsatz über vergleichbare Regelwerke in ausländischen Rechtsordnungen, der im Journal of Institutional and Theoretical Economics erschienen ist ${ }^{18}$.

Klaus Hopt stellt den Aufsichtsrat in den historischen Kontext. Dazu gehört auch die Konvergenz des amerikanischen und des deutschen Systems. Sie zeigt sich an der gewachsenen Bedeutung von independent directors im board amerikanische Unternehmen. Diese Entwicklung belegt er mit einem Aufsatz, der im Journal of Finance erschienen ist ${ }^{19}$.

\section{Das unausgeschöpfte Potential}

Die im dritten Abschnitt berichteten Texte verwenden offen sozialwissenschaftliche Argumente. Der natürliche nächste Schritt bestünde in der Validierung dieser Argumente. Wenn

14 W. Schön, Organisationsfreiheit und Gruppeninteresse im Europäischen Konzernrecht, Zeitschrift für Unternehmens-und Gesellschaftsrecht 48 (2019), 343-378, FN 34, s. auch FN 35 und FN 67.

15 B. Weitemeyer, Impulse der Stiftungsrechtsreform zur Governance und Transparenz von Stiftungen?, Zeitschrift für Unternehmens-und Gesellschaftsrecht 48 (2019), 238-270, FN26.

16 H. M. Anzinger, Vorstands-und Aufsichtsratsvergütung: Kompetenzverteilung und Offenlegung nach der zweiten Aktionärsrechterichtlinie, Zeitschrift für Unternehmens-und Gesellschaftsrecht 48 (2019), 39-96, FN 7; s. auch FN 155.

17 H. C. Grigoleit, Regulierung von Related Party Transactions im Kontext des deutschen Konzernrechts, Zeitschrift für Unternehmens-und Gesellschaftsrecht 48 (2019), 412-462, FN 5.

18 K. J. Hopt/P. C. Leyens, Der Deutsche Corporate Governance Kodex 2020, Zeitschrift für Unternehmensund Gesellschaftsrecht 48 (2019), 929-995, FN 83.

19 K. J. Hopt, Der Aufsichtsrat, Zeitschrift für Unternehmens-und Gesellschaftsrecht 48 (2019), 507-543, FN 37. 
das Argument in der sozialwissenschaftlichen Literatur bereits ausgearbeitet ist, könnte ein Rechtswissenschaftler die Ergebnisse berichten. Oft interessieren sich Sozialwissenschaftler aber nicht genau für das Argument, auf das es dem Juristen ankommt. Das gilt vor allem häufig für die Ausgestaltung von Institutionen. Wer etwas über die Bedeutung der Eingriffskondiktion für das Investitionsverhalten von Unternehmen lernen will, darf nicht mit Vorarbeiten der Ökonomen oder Psychologen rechnen. Auch wenn das juristisch relevante Argument in einer Sozialwissenschaft diskutiert ist, betrifft diese Diskussion doch meist nicht gerade die juristische Anwendung. Dann bleibt die Frage, ob die Einbettung in den juristisch relevanten Kontext den Effekt verändert. Diese Frage wird oft unter dem Gesichtspunkt der externen Validität diskutiert. Aus all diesen Gründen kann es lohnen, dass Juristen selbst sozialwissenschaftliche Methoden einsetzen. Im folgenden skizziere ich am Beispiel des Jahrgangs 2019 der ZGR, wie groß das unausgeschöpfte Potenzial ist.

Aus einer sozialwissenschaftlichen Perspektive sind Rechtsregeln Interventionen. Sie beschränken den Handlungsraum ihrer Adressaten. Das kann in der Absicht geschehen, normativ unerwünschtes Verhalten weniger wahrscheinlich zu machen. Die Beschränkung kann aber umgekehrt auch den Zweck verfolgen, normativ erwünschtes Verhalten wahrscheinlicher zu machen. Diese Ermöglichungsfunktion hat für das Gesellschaftsrecht besondere Bedeutung. In seinem Kern ist es ja Organisationsrecht. Es stellt der Wirtschaft einen Vorrat an Gestaltungsmöglichkeiten für die Selbstorganisation zur Verfügung.

Der corporate governance code ist bekanntlich kein zwingendes Recht. Klaus Hopt und Patrick Leyens halten inn dennoch für wirksam, weil der „in § 161 AktG niedergelegte[...] Grundsatz ,comply or explain' einen Anreiz zur Befolgung" setzt ${ }^{20}$. "Die deutschen börsennotierten Gesellschaften wollen Abweichungsbegrundungen möglichst vermeiden, wie aus der Praxis zu hören und wie die hohen Befolgungsquoten nahelegen" ${ }^{21}$. Es wäre reizvoll, diese Wirkbeziehung theoretisch genauer auszuarbeiten (wofür wäre solch eine Begründung am Kapitalmarkt ein Signal?) und den kausalen Effekt zu belegen.

Christian Decher setzt sich kritisch mit der Rechtsprechung des Bundesgerichtshofs auseinander, die den Bezugsrechtsausschluss in der Aktiengesellschaft einschränkt. Der Kern seiner Kritik ist funktional:

“Damit war das genehmigte Kapital seiner gesetzlich gedachten Funktion beraubt, der Gesellschaft bei sich bietenden Möglichkeiten schnell und flexibel neues Eigenkapital zur Verfügung zu stellen“22.

Aber hat die gerichtliche Kontrolle des Hauptversammlungsbeschlusses am Maßstab der Verhältnismäßigkeit auch wirklich diese Wirkung? Und in welchem Verhältnis steht die potenziell beschränkende Wirkung zu ermöglichenden Vorwirkungen? Wenn Anleger weniger Sorge darum haben müssen, dass das Bezugsrecht später ausgeschlossen wird, könnten

20 K. J. Hopt/P. C. Leyens, Der Deutsche Corporate Governance Kodex 2020, Zeitschrift für Unternehmensund Gesellschaftsrecht 48 (2019), 929-995, $930 f$.

21 Ebda. 947.

22 C. E. Decher, Von der Kontrolle des Bezugsrechtsausschlusses nach Kali+ Salz zur (eingeschränkten) Kontrolle nach allgemeinen Schranken?, Zeitschrift für Unternehmens-und Gesellschaftsrecht 48 (2019), 1122-1170, 1132. 
sie eher bereit sein, Aktien zu erwerben. Die widerstreitenden Effekte könnte man modellieren und empirisch testen.

Heribert Anzinger fragt, ob die Veröffentlichung der Vorstandsbezüge eine dämpfende Wirkung hat. "Vorstandsmitglieder könnten die öffentliche Wahrnehmung überzogener Gehälter zur Zurückhaltung motivieren"23. Es würde sich lohnen, die Kanäle genauer auszuarbeiten, auf denen dieser Effekt eintreten könnte. Dazu braucht man zunächst eine Vorstellung über handlungsleitende Motive. Geht es den Vorstandsmitgliedern nur oder jedenfalls maßgeblich um Geld? Dafür könnte Selektion eine Rolle spielen. Auch das Risiko, die gut bezahlte Aufgabe schnell zu verlieren, könnte Wirkung tun. Wenn das Einkommen das maßgebliche Motiv ist, kommt es auf die Möglichkeit von Einkommenseffekten an. Im Unternehmen könnte die Bereitschaft von Arbeitnehmern leiden, sich für das Unternehmen einzusetzen. Das könnte den Gewinn und in der Folge den Bonus schmälern. Öffentlicher Druck könnte den Aufsichtsrat motivieren, das Vorstandsmitglied abzuberufen.

Patrick Leyens hat Zweifel, ob die Information der Aktionäre in der Hauptversammlung einen legitimierenden Effekt hat:

„Erstens findet kein echter Austausch statt, weil uber die Berechtigung des Auskunftsverlangens und den Umfang der zu erteilenden Information unter großer Eile zu entscheiden ist, die Antworten zur Vermeidung von Anfechtungsklagen von einem back office nur mit dem Mindestinhalt versehen und sodann vom Versammlungsleiter letztlich allein zur Erfullung einer gesetzlichen Pflicht verlesen werden. Zweitens ist eine Informationsverarbeitung und echte Meinungsbildung der Aktionäre zu komplexen Entscheidungssachverhalten wie Strukturentscheidungen nicht ernsthaft ad hoc zu erhoffen. Dazu wäre eine komplexe Abwägung zwischen alternativen Möglichkeiten von Kurz-, Mittel- und Langfristerträgen des Gesellschaftskapitals Voraussetzung"24.

Das ist nicht unplausibel. Aber die Notwendigkeit zur expliziten Information der Hauptversammlung könnte den Kapitalmärkten bei der Neubewertung des Unternehmens helfen. Letztlich wäre eine genauere Wirkungsanalyse erforderlich, die idealerweise auch empirisch wäre.

Die Aktionärsrechterichtlinie ist besorgt, dass die Organe einer Aktiengesellschaft nicht wirksam kontrolliert werden, weil die Aktionäre überhaupt nicht informiert sind. Deshalb verpflichtet die Richtlinie die Mitgliedstaaten, ein Verfahren zur Identifikation der Aktionäre einzuführen. Dirk Zetzsche vergleicht Optionen zur Umsetzung dieser Verpflichtung ${ }^{25}$. Diese technischen Überlegungen sind wichtig. Aber sie bleiben auf Distanz zu dem norma-

23 H. M. Anzinger, Vorstands-und Aufsichtsratsvergütung: Kompetenzverteilung und Offenlegung nach der zweiten Aktionärsrechterichtlinie, Zeitschrift für Unternehmens-und Gesellschaftsrecht 48 (2019), 39-96, 52.

24 P. C. Leyens, System der Aktionärsinformation, Zeitschrift für Unternehmens-und Gesellschaftsrecht 48 (2019), 544-587, 557.

25 D. Zetzsche, Know Your Shareholder, der intermediärsgestützte Aktionärsbegriff und das Hauptversammlungsverfahren, Zeitschrift für Unternehmens-und Gesellschaftsrecht 48 (2019), 1-38. 
tiven Anliegen. Welche Unternehmens- und Konzernstruktur macht die Kontrolle der Unternehmensleitung besonders schwierig? Genügt der vorgeschlagene Eingriff, die Kontrolle effektiv zu machen?

Die Aktionärsrechterichtlinie verpflichtet die Mitgliedstaaten der Europäischen Union, das Verhalten von Stimmrechtsberatern zu regulieren. Martin Schockenhoff und Anton Nußbaum analysieren den Regelungsbedarf. Herrschaft (Einfluss auf unternehmerische Entscheidungen) und Haftung (ein Wertverlust der Aktien) können auseinanderfallen ${ }^{26}$, weil Aktionäre keinen Anreiz haben, die Empfehlungen der Berater zu überprüfen. ${ }^{27}$ Es gibt Interessenkonflikte, wenn der Stimmrechtsberater nicht nur die Inhaber von Stimmrechten berät, sondern auch die Unternehmensleitung ${ }^{28}$. Die abgestimmte Beratung der Aktionäre von Konkurrenten kann ein Kartell ersetzen ${ }^{29}$. All das ist Stoff für ökonomische Modelle.

Walter Doralt sorgt sich, dass der diszipliniere Effekt der Organhaftung durch D\&O Versicherungen konterkariert wird.

„Die Reaktion des Gesetzgebers blieb daher ein etwas halbherziger Kompromiss und beschränkte sich auf die Einfuhrung eines - wirtschaftlich gut tragbaren, aber doch spurbaren - zwingenden Selbstbehalts in § 93 Abs. 2 Satz 3 AktG" ${ }^{\prime 30}$.

Das ist eine testbare Hypothese: wenn Vorstandsmitglieder nicht den vollen Schaden begleichen müssen, den die Gesellschaft erlitten hat, haben sie einen geringeren Anreiz, das Gesellschaftsinteresse zu verfolgen. Das muss nicht richtig sein. Das normative Ziel ist ja nicht Kompensation, sondern Verhaltenssteuerung. Der Schaden aus unternehmerischen Fehlentscheidungen wird häufig so groß sein, dass das Vorstandsmitglied sein gesamtes Vermögen verlöre, wenn es dafür aufkommen müsste. Der Effekt könnte sogar kontraproduktiv sein und Vorstandsmitglieder zu waghalsigen Entscheidungen motivieren; wenn die Sache schief geht, hätten sie ohnehin ihre bürgerliche Existenz verloren.

Nach § 93 II 1 AktG haften die Mitglieder des Vorstands einer Aktiengesellschaft gegenüber der Gesellschaft für einen Schaden, der aus der Verletzung ihrer Pflichten entsteht. Der Bundesgerichtshof hat nun entschieden, dass der bloße Nachweis eines Kompetenzverstoßes noch keine Haftung begründet. Damit hat er den Einwand des rechtmäßigen Alternativverhaltens eröffnet. Maximilian Goette begrüßt diese Entscheidung: „der II. Zivilsenat [eröffnet] hiermit in Anspruch genommenen Vorständen eine weitere Verteidigungsmöglichkeit $^{31}$. Die alternativen Interpretationen der Norm könnte man modellieren und dann besser abschätzen, welche Wirkungen auf die Erhebung einer Klage zu erwarten sind.

26 S. erneut A. A. Berle/G. C. Means, Modern Corporation and Private Property (1932).

27 M. Schockenhoff/A. Nußbaum, Die neuen Transparenzvorschriften für Stimmrechtsberater, Zeitschrift für Unternehmens-und Gesellschaftsrecht 48 (2019), 163-190, 169.

28 Ebda.

29 Ebda. 170.

30 W. Doralt, Organhaftung und D\&O-Versicherung, Zeitschrift für Unternehmens-und Gesellschaftsrecht 48 (2019), 996-1049, 1001.

31 M. Goette, Aufsichtsrats-und Hauptversammlungsbeschluss-zugleich Urteilsbesprechung zu BGH II ZR 24/17, Zeitschrift für Unternehmens-und Gesellschaftsrecht 48 (2019), 324-338, 331. 
Jens Ekkenga fragt sich, ob die Macht des Minderheitsgesellschafters einer $\mathrm{GmbH}$ beschränkt werden muss, wenn diese Macht allein darauf beruht, dass der Mehrheitsgesellschafter nach § 47 IV GmbHG von der Abstimmung ausgeschlossen ist. Er plädiert dafür, dass der Minderheitsgesellschafter keine verbandsschädlichen Entscheidungen treffen darf, und dass ihn dafür die Beweislast trifft ${ }^{32}$. Es würde sich lohnen, den Vorwirkungen dieser Umkehr der Beweislast auf die Entscheidungen des Minderheitsgesellschafters nachzugehen. Wird dadurch der Schutz der Minderheit beseitigt, den das Gesetz wegen eines Interessenkonflikts des Mehrheitsgesellschafters gewünscht hat?

Klaus Hopt wendet sich gegen die These, der Aufsichtsrat dürfe nicht am Vorstand vorbei Fragen an die Belegschaft richten. Andernfalls drohe ein Verlust der Autorität des Vorstands ${ }^{33}$. Wollte man es genau wissen, müsste man Wirkungskanäle spezifizieren. Muss der Vorstand eine tiefer wirkende Kontrolle durch den Aufsichtsrat gewärtigen? Das wäre vermutlich im Sinne des Gesetzes. Geht es um die Sorge, dass oppositionelle Strömungen im Unternehmen den Aufsichtsrat auf ihre Seite ziehen? Zumindest bei mitbestimmten Unternehmen wäre auch das wohl vom Willen des Gesetzes gedeckt. Oder ist man besorgt, dass der Vorstand auf Entscheidungen verzichtet, um dem Aufsichtsrat keine Gelegenheit zur Intervention zu bieten? Das wäre vermutlich nicht mehr im Sinne des Gesetzes.

Die unternehmerische Mitbestimmung ist bekanntlich eine Besonderheit des deutschen Rechts. Das Europäische Gerichtshof hatte über eine Folgefrage zu entscheiden. Ist es mit der Freizügigkeit der Arbeitnehmer zu vereinbaren, wenn ein Arbeitnehmer das aktive und passive Wahlrecht für die Wahl der Arbeitnehmervertreter im Aufsichtsrat verliert, wenn er von einer inländischen zu einer ausländischen Tochter wechselt? Er verliert dann gegebenenfalls auch sein Aufsichtsratsmandat. Achim Seifert billigt die Entscheidung im Ergebnis, kritisiert aber, dass der Gerichtshof kein Kriterium für eine „Beschränkung" der Freizügigkeit entwickelt hat ${ }^{34}$. Es hätte nahe gelegen, die Vorwirkungen dieser Auslegung zu untersuchen. Der Verlust des aktiven Wahlrechts wird schwerlich so viel Gewicht haben, dass ein Arbeitnehmer auf die Tätigkeit in einem anderen Mitgliedstaat verzichtet. Der Verlust des Aufsichtsratsmandats dürfte aber einen "chilling effect" haben.

Die Aktionärsrechterichtlinie schreibt vor, dass „related party transactions“ geregelt werden. Hans Christoph Grigoleit behauptet: „Jedoch fällt der „zusätzliche“ Schutzeffekt der neuen Regulierung bei näherer Betrachtung nicht wesentlich ins Gewicht" ${ }^{35}$. Für den Nachweis bräuchte man sozialwissenschaftliche Methoden. Man müsste zunächst operationalisieren, welchen Gefahren möglicherweise wirksamer begegnet wird als mit den hergebrachten Mitteln des Konzernrechts. Der empirische Nachweis wäre schwierig, weil die Regeln der Richtlinie und ihre Umsetzung in das deutsche Recht breit diskutiert worden sind.

32 J. Ekkenga, Stimmverbote gegen den herrschenden Gesellschafter im GmbH-Konzern, Zeitschrift für Unternehmens-und Gesellschaftsrecht 48 (2019), 191-237, 237.

33 K. J. Hopt, Der Aufsichtsrat, Zeitschrift für Unternehmens-und Gesellschaftsrecht 48 (2019), 507-543, 529.

34 A. Seifert, Rezension zu EuGH, Urteil vom 18. Juli 2017, C-566/15 (Konrad Erzberger/TUI AG), Zeitschrift für Unternehmens-und Gesellschaftsrecht 48 (2019), 702-716, 707.

35 H. C. Grigoleit, Regulierung von Related Party Transactions im Kontext des deutschen Konzernrechts, Zeitschrift für Unternehmens-und Gesellschaftsrecht 48 (2019), 412-462, 413. 
Deshalb kann man nicht einfach die Ergebnisse vor und nach der Änderung der Regeln vergleichen.

Hans-Friedrich Müller konzentriert sich auf die Weise, mit der der deutsche Gesetzgeber diese Verpflichtung der Richtlinie umsetzen will. Dazu gehört die in § 48a I 1 WpHG-E vorgesehene Publizität von Geschäften mit nahestehenden Unternehmen oder Personen. Die Veröffentlichung muss zwar unverzüglich, aber erst nach Abschluss des Geschäfts erfolgen. „Immerhin können aber auch ex-post zur Verfügung gestellte Informationen die Erhebung einer Schadensersatzklage der Aktionäre erleichtern und für potentielle Investoren Grundlage ihrer Anlageentscheidung sein" ${ }^{36}$. Es wurde lohnen, diese beiden Kanäle genauer auszuarbeiten.

Es ist eigentlich nicht die Aufgabe eines Richters beim Bundesgerichtshof, in Eigeninitiative das Recht fortzuentwickeln. Ein ehemaliges Mitglied des II. Zivilsenats hat diesen Versuch jedoch unternommen. Zum Schutz der Aktionäre einer verschmelzenden Gesellschaft soll nach dem sog. Linde/Praxair-Verfahren genügen, wenn ihnen ein Umtauschangebot für ihre Aktien gemacht wird. Lutz Strohn fordert stattdessen einen Hauptversammlungsbeschluss. Jens Koch prüft diesen Vorschlag ${ }^{37}$. Doch um die alternativen Lösungen wirklich vergleichen zu können, müsste man die Schutzeffekte modellieren (und idealerweise auch empirisch testen).

Peter Hommelhoff betont die Legitimität eines faktischen Konzerns als Organisationsform für arbeitsteiliges Wirtschaften ${ }^{38}$. Die Legitimität speist sich auch aus der „rechtlich induzierte[n] Handlungsdynamik" ${ }^{\prime 39}$. Das ist eine spannende These, die nach einem empirischen Test ruft. Führt der partielle rechtliche Schutz vor dem Einfluss der Konzernmutter tatsächlich zu größerer Autonomie der Konzerntöchter?

Wolfgang Schön fordert eine Neujustierung des Konzernrechts. Die Aufgabe dieses Rechtsgebiets beschränkt sich nicht auf den Schutz der Minderheit einer Konzerntochter vor dem Missbrauch der Leitungsmacht der Konzernmutter. Das Konzernrecht hat auch eine Ermöglichungsfunktion. Es soll der Mutter erlauben, Synergien zu nutzen. Die rechte Balance findet man, wenn man den Schutz der Minderheit im Sinne eines „dulde und liquidiere“ auf das Gewinninteresse beschränkt. ${ }^{40}$ Das ist ein spannender Vorschlag, der nach einem Modell ruft: welche Effekte sind im Gleichgewicht zu erwarten, wenn der Minderheit die Möglichkeit verwehrt wird, ihr nachteilige Leitungsentscheidungen abzuwehren? Würden dann Konzerne gar nicht mehr entstehen, deren Bildung effizient wäre? Könnte sich die Konzernmutter den wirtschaftlichen Wert einer Minderheitsbeteiligung in einer zuvor selbstständigen Tochter aneignen, weil die Minderheit aus dem Konzern gedrängt wird?

36 H.-F. Müller, Related Party Transactions im Konzern, Zeitschrift für Unternehmens-und Gesellschaftsrecht 48 (2019), 97-125, 105.

37 J. Koch, Die Zuständigkeit der Hauptversammlung für Zusammenschlussvorhaben nach dem Linde/Praxair-Modell, Zeitschrift für Unternehmens-und Gesellschaftsrecht 48 (2019), 588-629.

$38 P$. Hommelhoff, Konzernorganisation und Haftungsbeschränkung, Zeitschrift für Unternehmens-und Gesellschaftsrecht 48 (2019), 379-411, 390.

39 Ebda.

40 W. Schön, Organisationsfreiheit und Gruppeninteresse im Europäischen Konzernrecht, Zeitschrift für Unternehmens-und Gesellschaftsrecht 48 (2019), 343-378, 356 f. 
Holger Fleischer und Sofie Cools zeichnen die Geschichte des Personengesellschaftsrechts und seine Entwicklung in anderen Rechtsordnungen nach ${ }^{41}$. Der Text stellt keine funktionalen Thesen auf. Man könnte Konvergenz und Divergenz aber auch quantitativ analysieren. Interessant wäre zum Beispiel, ob machine learning algorithms die Rechtsfamilien finden, die die juristische Diskussion erwartet ${ }^{42}$.

Volker Beuthien will das Konzept der Gesamthand tiefer verstehen. Die Botschaft findet sich gleich auf der ersten Seite:

„Da sowohl die Personen der Gesamthänder als auch die Gegenstände eines Gesamthandvermögens beliebig sind, kann kennzeichnend furr eine Gesamthand als solche nur sein, dass die an ihr beteiligten Personen durch die Gemeinschaftlichkeit ihrer Rechtsinhaberschaft in der Rechtsausubung beschränkt sind, während das aus der gemeinsamen Verwaltung ihres Sondervermögens ergebende persönliche Rechtsverhältnis der Gesamthänder untereinander nicht Ursprung, sondern nur Folge der Rechtsgemeinschaft ist" ${ }^{43}$.

Normativ ist die Rechtsordnung natürlich frei, die Gesamthand aus der Perspektive der Vermögensbeziehungen zu konstruieren. Aber verfolgt die Rechtsordnung mit der Institution der Gesamthand nicht auch den Zweck, dem Rechtsverkehr geeignete Institutionen zur Verfügung zu stellen? Dann könnte es durchaus wichtig sein, die Institution so auszugestalten, dass enge persönliche Beziehungen entstehen, die die gemeinsam gewählte Aufgabe tragen können. Dazu hätte die Sozial- und Organisationspsychologie etwas zu sagen.

Christine Osterloh-Konrad hält das Gebot der Selbstorganschaft in der Personengesellschaft für überlebt. „An der überkommenen Vorstellung, das Fehlen einer persönlichen Haftung fuhre generell zu ubertrieben risikofreudiger Unternehmensfuhrung, scheint nicht viel dran zu sein"44. Das mag ja stimmen. Aber was ist falsch an der Sorge um das Auseinanderfallen von Herrschaft und Haftung ${ }^{45}$ ? Welche institutionellen Sicherungen sind an die Stelle getreten?

Nach § 172 IV HGB lebt die persönliche Haftung eines Kommanditisten wieder auf, wenn er Anteile entnommen hat und das Unternehmen so viel Verlust macht, dass der Wert seines Kapitalanteils unter den Betrag der geleisteten Einlage sinkt. Der Bundesgerichtshof hatte über eine prozessrechtliche Folgefrage zu entscheiden. Wie weit reicht die Darlegungslast des Kommanditisten im Rechtsstreit mit dem Insolvenzverwalter? Christoph Thole plädiert dafür, den normativen Konflikt offen zu legen: „Der Kommanditist trägt die Darlegungs- und

$41 \quad H$. Fleischer/S. Cools, Internationale Trends und Neuerungen im Personengesellschaftsrecht, Zeitschrift für Unternehmens-und Gesellschaftsrecht 2019, 463-506.

42 S. zum Vergleich Y.-c. Chang et al., Drawing the Legal Family Tree: An Empirical Comparative Study of

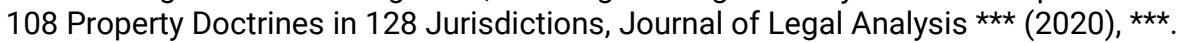

43 V. Beuthien, Die Gesamthand-was ist das eigentlich?, Zeitschrift für Unternehmens- und Gesellschaftsrecht 48 (2019), 664-701, 665.

44 C. Osterloh-Konrad, Die Selbstorganschaft in der Personengesellschaft-Wesenszug oder Anachronismus?, Zeitschrift für Unternehmens-und Gesellschaftsrecht 48 (2019), 271-300, 296.

45 S. erneut A. A. Berle/G. C. Means, Modern Corporation and Private Property (1932). 
Beweislast furr die fehlende Erforderlichkeit zur Gläubigerbefriedigung"46. Die Analyse der resultierenden Anreize wäre spannend. Wie würde sich ein auf die Maximierung seines Einkommens zielender Kommanditist verhalten? Würde er bereits auf die Entnahme von Gewinnen verzichten? Oder gäbe es sogar Vorwirkungen auf die Entscheidung, sich als Kommanditist an einer Personengesellschaft zu beteiligen? Wären solche Vorwirkungen normativ als Selektionsinstrument erwünscht?

Nach § 116 II HGB bedürfen außergewöhnliche Entscheidungen in einer Personengesellschaft der Zustimmung aller Gesellschafter. Der Bundesgerichtshof hatte über die Wirksamkeit einer Satzungsklausel zu entscheiden, die auch für solche Entscheidungen eine qualifizierte Mehrheit vorschreibt. Das hat der Bundesgerichtshof gebilligt, weil § 116 II HGB dispositives Recht sei. Herbert Wiedemann hält die Entscheidung für richtig:

„In einer Publikumsgesellschaft, an der sich eine große Zahl untereinander (zunächst) nicht verbundener, geschweige denn organisierter Kapitalanleger beteiligen, muss der ,Gesellschaftswille' von der Stimmenmehrheit gebildet werden, weil Vertragsänderungen sonst nicht durchzubringen wären, selbst wenn sie offenkundig im Interesse des Gemeinwohls liegen“" ${ }^{47}$.

Die Analyse der Anreizeffekte wäre interessant. Welche Vorwirkungen auf die Anlageentscheidung hat die resultierende Unsicherheit über den Gegenstand und damit die Werthaltigkeit der Anlage? Unterscheidet sich die Unsicherheit, die aus künftigen Entscheidungen einer dann bestehenden Mehrheit resultiert, von der Unsicherheit über den Erfolg des Unternehmens am Markt? Letztere Unsicherheit ist mit jeder Beteiligung an einem Unternehmen verbunden.

Stefan Korch interpretiert eine Vorschrift der Restrukturierungsrichtlinie als legislative Definition des Unternehmenszwecks. Wenn das Unternehmen in eine Krise gerät, ist der Geschäftsleiter nach Art. 19 verpflichtet, die Interessen der Gläubiger, der Anteilseigner sowie weiterer stakeholder in einen angemessenen Ausgleich zu bringen ${ }^{48}$. Eine sozialwissenschaftliche Folgefrage liegt nahe: welche verhaltenslenkenden Effekte haben gesetzliche Definitionen des Unternehmenszwecks?

Darf der Alleingesellschafter einer $\mathrm{GmbH}$ das Unternehmen dadurch sanieren, dass er es mit einer zweiten $\mathrm{GmbH}$ verschmilzt, an der er die Mehrheit hält? Der Bundesgerichtshof hat die Frage für den Fall verneint, dass dadurch die Existenz der aufnehmenden Gesellschaft vernichtet wird. Jan Lieder und Martin Bialluch fordern außerdem eine Differenzhaftung: Daran „fuhrt schon aus materiellen Gerechtigkeitserwägungen kein Weg vorbei: Wird

46 C. Thole, Die Wirkungen der Feststellung von Gläubigerforderungen zur Insolvenztabelle gegenüber dem nach $\S \S 171,172$ Abs. 4 HGB haftenden Kommanditisten, Zeitschrift für Unternehmens-und Gesellschaftsrecht 48 (2019), 301-323, 310.

47 H. Wiedemann, Mehrheitsbeschlüsse und Geschäftsführerhaftung in der GmbH \& Co. KG, Zeitschrift für Unternehmens-und Gesellschaftsrecht 48 (2019), 923-928, 924.

48 S. Korch, Sanierungsverantwortung von Geschäftsleitern, Zeitschrift für Unternehmens- und Gesellschaftsrecht 48 (2019), 1050-1081. 
ein neuer Anteil an den Inferenten ausgegeben, erfordert das Gebot eines effektiven Kapitalschutzes, dass die erbrachte Einlage werthaltig ist“49. „Gerechtigkeitserwägungen“ sind ein wenig klarer normativer Maßstab. Geht es um Verteilungsgerechtigkeit? Aber müsste dann nicht jeder Konzern verboten werden? Oder geht es um Anreizeffekte? Würden normativ erwünschte Konzerne nicht mehr gebildet, wenn die Rechtsordnung diesen Schutz verwehrt? Oder geht es um den Schutz des Schwächeren? Aber ist jeder Minderheitsgesellschafter von vornherein der Macht des Mehrheitsgesellschafters ausgeliefert? Warum hat er sich dann an dem Unternehmen beteiligt?

York Schnorbus plädiert für die Zulässigkeit einer Direktabspaltung als Gestaltungsform für einen Spin-Off. Die bisherige Konzernmutter gibt die Anteile an der bisherigen Tochter auf. Aktionäre der Mutter erwerben diese Anteile. Der Spaltungsfreiheit soll aber die gesamtschuldnerische Haftung von Mutter und Tochter für die Altschulden der Mutter korrespondieren. Andernfalls ginge den Gläubigern der Mutter Haftungsmasse verloren ${ }^{50}$. Dieses Argument lässt sich als Anwendung eines schon mehrfach verwendeten Kerngedankens der ökonomischen Analyse des Rechts interpretieren: Herrschaft (in diesem Fall: die Freiheit der bisherigen Konzernmutter zur Auflösung der Konzernbeziehung) und Haftung sollen gleichlaufen ${ }^{51}$.

Jannik Otto kritisiert die Rechtsprechung des Bundesgerichtshofs zur Schiedsfähigkeit von Streitigkeiten über Mängel gesellschaftsrechtlicher Beschlüsse.

„Die Rechtsordnung geht [...] im Ausgangspunkt von einer grundsätzlichen Gleichwertigkeit der Streitbeilegung im Schiedsverfahren mit der Streitbeilegung vor staatlichen Gerichten aus. [...] Eine Benachteiligung einer Partei kann sich also nur aus hinzutretenden Umständen ergeben. Die Wirksamkeitsprufung gem. § 138 Abs. 1 BGB ist daher auf die Umstandskontrolle beschränkt" ${ }^{52}$.

Es ist natürlich legitim, normative Fragen positivistisch zu entscheiden: der Gesetzgeber hat die Verantwortung übernommen. Der Interpret will sich nicht über diese Entscheidung hinwegsetzen. Diese Art von „academic self-restraint“ ist jedoch selten geworden. Normalerweise möchte ein Rechtswissenschaftler die Entscheidung des Gesetzgebers nicht nur berichten, sondern auch verstehen. Dann wäre die Frage berechtigt, ob Schiedsverfahren für diese Klasse von Streitigkeiten wirklich gleich geeignet sind. Das könnte eine Wirkungsanalyse zeigen, für die man sozialwissenschaftliche Methoden bräuchte.

49 J. Lieder/M. Bialluch, Differenzhaftung und Existenzvernichtungshaftung bei Verschmelzung, Zeitschrift für Unternehmens-und Gesellschaftsrecht 48 (2019), 760-779, 764.

50 Y. Schnorbus, Die Direktabspaltung, Zeitschrift für Unternehmens-und Gesellschaftsrecht 48 (2019), 780$812,788$.

51 S. noch einmal A. A. Berle/G. C. Means, Modern Corporation and Private Property (1932).

$52 \mathrm{~J}$. Otto, Anforderungen an die Schiedsvereinbarung für gesellschaftsrechtliche Beschlussmängelstreitigkeiten, Zeitschrift für Unternehmens-und Gesellschaftsrecht 48 (2019), 1082-1121, 1087. 


\section{Korrelationen}

Kausalität ist ein anspruchsvolles Konzept. Ob ein bestimmter Gegenstand, eine bestimmte literarische Gattung, eine bestimmte Ausbildung oder ein bestimmtes Wirkungsfeld ursächlich dafür ist, dass ein Autor sozialwissenschaftliche Methoden oder Ergebnisse rezipiert, lässt sich aus der Durchsicht eines Jahrgangs einer Zeitschrift nicht belegen. Andere Ursachen könnten entscheidend sein, die so nicht zu beobachten sind. Vor allem ist aber die Richtung der Kausalität unklar. Sucht sich der Gegenstand seine Methode, oder motiviert die Eignung zur Anwendung einer Methode die Wahl des Gegenstands? Hat ein Autor auf die Rezeption sozialwissenschaftlicher Erkenntnisse verzichtet, weil sich eine Urteilsanmerkung dafür nicht eignet, oder hat er sich für das Schreiben einer Anmerkung entschlossen, weil ihm die sozialwissenschaftlichen Erkenntnisse nicht zugänglich sind? Benutzt ein Autor sozialwissenschaftliche Literatur, weil er dafür die Ausbildung hat, oder hat er die Ausbildung erworben, um diese Literatur nutzen zu können? Berücksichtigt ein Autor sozialwissenschaftliche Ergebnisse, weil er das als Universitätsprofessor für seine Pflicht hält, oder ist er Professor geworden, damit er seine Aussagen auch interdisziplinär absichern kann?

Die folgende Tabelle berichtet deshalb nur Korrelationen. Zwei Variablen stehen in einem Zusammenhang. Wenn die Wahrscheinlichkeit sehr klein ist, dass dieser Zusammenhang auf Zufall beruht, lohnt es, den Zusammenhang näher zu untersuchen. Die Konvention der Sozialwissenschaften wird als p-Wert ausgedrückt. Bei kleinen Datensätzen wie diesem gilt ein $p$-Wert unter 0,05 als bedeutsam, und ein $p$-Wert unter 0,1 als berichtenswert.

\begin{tabular}{|c|c|c|c|c|c|c|c|}
\hline & $\begin{array}{l}\text { Modell } \\
1\end{array}$ & $\begin{array}{l}\text { Modell } \\
2\end{array}$ & $\begin{array}{l}\text { Modell } \\
3\end{array}$ & $\begin{array}{l}\text { Modell } \\
4\end{array}$ & $\begin{array}{l}\text { Modell } \\
5\end{array}$ & $\begin{array}{l}\text { Modell } \\
6\end{array}$ & $\begin{array}{l}\text { Modell } \\
7\end{array}$ \\
\hline $\begin{array}{l}\text { Corporate } \\
\text { Governance }\end{array}$ & $\begin{array}{l}0,640 \\
(0,066)\end{array}$ & & & & $\begin{array}{l}0,560 \\
(0,093)\end{array}$ & $\begin{array}{l}0,629 \\
(0,058)\end{array}$ & $\begin{array}{l}0,716 \\
(0,034)\end{array}$ \\
\hline Anmerkung & & $\begin{array}{l}-0,667 \\
(0,041)\end{array}$ & & & $\begin{array}{l}-0,600 \\
(0,059)\end{array}$ & $\begin{array}{l}-0,543 \\
(0,081) \\
\end{array}$ & $\begin{array}{l}-0,435 \\
(0,168) \\
\end{array}$ \\
\hline $\begin{array}{l}\text { sozialwissen- } \\
\text { schaftliche } \\
\text { Ausbildung }\end{array}$ & & & $\begin{array}{l}0,440 \\
(0,187)\end{array}$ & & & $\begin{array}{l}0,457 \\
(0,139)\end{array}$ & $\begin{array}{l}0,394 \\
(0,201)\end{array}$ \\
\hline Prof & & & & $\begin{array}{l}0,593 \\
(0,154)\end{array}$ & & & $\begin{array}{l}0,513 \\
(0,201)\end{array}$ \\
\hline cons & $\begin{array}{l}-0,000 \\
(1,000)\end{array}$ & $\begin{array}{l}0,667 \\
(0,000)\end{array}$ & $\begin{array}{l}0,417 \\
(0,012)\end{array}$ & $\begin{array}{l}-0,000 \\
(1.000)\end{array}$ & $\begin{array}{l}0,200 \\
(0,517)\end{array}$ & $\begin{array}{l}0,029 \\
(0,929)\end{array}$ & $\begin{array}{l}-0,499 \\
(0,337)\end{array}$ \\
\hline
\end{tabular}

Table 1

abhängige Variable: 2: intensive Auseinandersetzung mit sozialwissenschaftlicher Literatur, 1: einzelne

Zitate, 0: keine Zitate

lineare Modelle

$\mathbf{N}=\mathbf{3 1}$

p-Werte in Klammern 
Auch wenn es nur Korrelationen sind, sind die Ergebnisse interessant. Alle sieben Modelle korrelieren das Ausmaß, in dem ein Aufsatz sozialwissenschaftliche Literatur auswertet, mit beobachteten Eigenschaften des Gegenstands, der literarischen Gattung oder des Autors. Modelle 1-4 korrelieren die Auswertung sozialwissenschaftlicher Literatur mit nur je einer anderen Variablen. Dass der Autor eine wirtschaftswissenschaftliche Ausbildung hat und dass er ein Professor ist, hat zwar den erwarteten positiven Effekt. Die p-Werte sind aber weit von dem konventionellen Maß für die Signifikanz eines Effekts entfernt. Das mag daran liegen, dass die 31 Aufsätze des Jahrgangs 2019 ein zu kleines Sample sind. Große Bedeutung haben diese beiden Faktoren aber jedenfalls nicht. Blickt man nur auf bivariate Korrelationen, ist die literarische Gattung am wichtigsten. Modell 2 zeigt nicht nur einen klar signifikanten, negativen Effekt $(p=0,041)$. Tatsächlich addieren sich die Konstante und der Koeffizient für den Umstand, dass der Aufsatz eine Urteilsanmerkung ist, zu 0 . In keiner der sieben Urteilsanmerkungen findet sich ein einziges sozialwissenschaftliches Zitat. Dass der Aufsatz eine Frage der corporate governance zum Gegenstand hat, hat einen deutlich positiven Effekt. Auch in Modell 1 lohnt der Blick auf die Konstante. Sie ist exakt 0. Sozialwissenschaftliche Literatur wird im Jahrgang 2019 der ZGR nur zitiert, wenn es um corporate governance geht. Dieser Effekt hat allerdings einen p-Wert von 0,066. Er ist nur „schwach signifikant“. Die bivariate Korrelation ist nur bedingt verlässlich.

Modelle 5-7 „kontrollieren“ dann aber für mehrere der beobachteten Variablen. Das ändert die Interpretation der Koeffizienten. Sie sind nun konditioniert darauf, dass die übrigen Variablen 0 sind. Am Beispiel von Modell 5: wenn der Aufsatz keine Urteilsanmerkung ist, dann wird weniger wichtig, ob er corporate governance zum Thema hat (der Koeffizient ist kleiner als in Modell 1, der p-Wert ist größer). Umgekehrt wird auch weniger wichtig, dass der Text ein Urteil kommentiert, wenn es dabei nicht um corporate governance geht (der Koeffizient ist kleiner als in Modell 2, der p-Wert ist größer). Diese Unterschiede zwischen Modellen 1 und 2 auf der einen und Modell 5 auf der anderen Seite beruhen darauf, dass beide Variablen negativ korreliert sind. Urteilsanmerkungen widmen sich häufiger Gegenständen außerhalb von corporate governance.

Modelle 6 und 7 „kontrollieren“ zusätzlich noch für Charakteristika des Autors. Tut man das, erweist sich, dass der Gegenstand wichtiger ist als die literarische Gattung. In Modell 7 ist der Koeffizient für den Gegenstand corporate governance am größten von allen Modellen. Der Koeffizient dafür, dass der Text ein Urteil kommentiert, ist am kleinsten. Mit den p-Werten verhält es sich genau umgekehrt.

\section{Entwicklungsoptionen}

Die deutsche Rechtswissenschaft ist zu Recht stolz auf ihre Nähe zur Rechtspraxis. Rechtswissenschaft will zuerst einmal nützlich sein. Sie dient den Rechtsunterworfenen, weil sie Gerichten, Behörden und Vertragsparteien hilft, bessere Entscheidungen zu treffen. Das gilt auch für das Gesellschaftsrecht. Der amerikanischen Rechtswissenschaft werfen deutsche Wissenschaftler nicht selten vor, dass sie diese Verbindung zur Rechtspraxis gekappt hat. Wissenschaftlichkeit ist für einen Juristen kein Selbstzweck. Trotzdem hoffe 
ich, dass diese Spurensuche belegt, wie viel unmittelbaren Nutzen gerade die Rechtspraxis ziehen könnte, wenn sich das Gesellschaftsrecht noch stärker auf die sozialwissenschaftliche Literatur einlässt. Am Ende wird sich die Disziplin dann auch dafür öffnen müssen, selbst sozialwissenschaftliche Methoden anzuwenden. Denn was der Rechtspraxis dient, weiß ein Rechtswissenschaftler viel besser und genauer als sein Kollege, der vor allem das wissenschaftliche Gespräch zwischen Wirtschaftswissenschaftlern, Psychologen oder Soziologen sucht. 